\title{
Pion Mass Dependence of Nucleon Magnetic Moments
}

\author{
F. L. Braghin \\ Instituto de Física da Universidade de São Paulo, C.P. 66.318, CEP 05315-970, São Paulo, SP Brasil
}

Received on 15 August, 2003.

\begin{abstract}
The relevance of the pion mass, provenient from a term which explicitely breaks chiral symmetry in the Lagrangian, for nucleon magnetic moment in the frame work of the Skyrme model in two different versions: the usual one model and a modified one which includes a coupling to a light scalar meson field, the sigma $\sigma(\simeq 500-600 \mathrm{MeV})$. Results are compared to other calculations. Our main motivation comes from usual extrapolations for values of low energy QCD observables obtained in lattices with large values of pion/quark masses toward realistic value of $m_{\pi}$ which do not allow it. We do a comparison with results from the Cloudy Bag Model and a chiral hadronic model from chiral perturbation theory. There are several resulting extrapolations from the region of large pion mass to the realistic value depending on the considered model for low energy QCD.
\end{abstract}

\section{Introduction}

Quantum Chromodynamics is a very intrincate theory with flavor and color non abelian structure and strong coupling constants at low energies. There are basically two ways of extracting physical observables from QCD. Lattice calculations in discretized space-time system and effective models with the relevant hadronic, quark and gluon degrees of freedom considering the more fundamental properties and symmetries. In most of QCD lattice calculations it is not possible to obtain full reliable results mainly because the lattices can not be large enough and it does not provide realistic values for quark-(pion) masses [1,2]. Therefore it is needed to perform extrapolations from exact calculations in finite lattice to the physical region of observables to ensure reliability. For this task one can make use of effective models for the description of low and intermediary energy QCD. They are based on general properties, symmetries and their dynamical breakdowns of the believed more fundamental theory, QCD. The non linear sigma model with pions, and extensions which gave origin to Chiral Perturbarion Theory (CPT), is obtained from the linear realization by fixing the fourth degree of freedom, the sigma field. This is possible due to the dynamical chiral symmetry breaking expected to be realized in the Nambu mode. Recently there has been claimed that a scalar sigma particle $(\sigma(\simeq 500-600 \mathrm{MeV})$ exists $[3,4,5,6,7])$. However such field, that may develop a classical counterpart (the chiral condensate of the chiral dynamical breaking), has been neglected in many kinds of calculations including lattice calculations. Therefore it becomes very interesting to study the effect of such "mesonic" degree of freedom at the phenomenological and theoretical levels like it has been done in the Skyrmion model and other models $[3,8]$. In particular in $[3,4,9,10]$ it is shown that this coupling may lead to a redefinition of the chiral radius (which may become a coordinate dependent function), which can be different from the classical value of the sigma field (or equivalently the scalar quark-anti-quark condensate). The quark masses, and also the pion masses, are expected to break Chiral symmetry explicitly appearing in Lagrangian terms ${ }^{1}$. An expected relation between these variables, of QCD and hadronic degrees of freedom, can be seen through the Gell-Mann-Oakes-Renner relation which, to the lowest order, reads:

$$
2<\bar{q} q>m_{q}=-f_{\pi}^{2} m_{\pi}^{2}
$$

Therefore one is to consider that large pion mass is, in principle, equivalent to large quark mass which break, both, chiral symmetry ${ }^{2}$ In lattice calculations the realistic value for these masses are not use and the large values are useful for providing first estimations to be extrapolated to the correct values.

In this work we want to address the subject of these extrapolations done for lattice calculations by considering different large pion masses for the calculation of nucleon static properties, in particular their magnetic moments, by using two different versions of the Skyrmion model. The results are compared to previous calculations. The Adelaide group [1] used the cloudy bag model for giving such a extrapolation for the dependence of the nucleons magnetic moments on the pion mass. This has been object of attention also of [2] which considered chiral perturbation theory. It is shown that there are different possibilities of extrapolating results from the region in which pion mass is large to the chiral limit depending on the model and its physical content.

\footnotetext{
${ }^{1}$ Descriptions and origins for these masses are proposed respectively in [9].

${ }^{2}$ Alternative proposal may be envisaged $[9,12]$.
} 


\section{The Skyrme model and extension with scalar meson field}

\subsection{The Skyrmion}

The Skyrmion model has been extensively discussed in the literature therefore we omit details about it. The Skyrmion model is basically a solitonic solution of the non linear sigma model with a non trivial topology. The usual non linear sigma model with the pion field alone however does not possess stable solitonic solutions and a stabilizing term with higher order derivatives (usually fourth order) is introduced as proposed by Skyrme long ago [13] - sixth and higher order derivative terms may also be considered - see for example [14]. These fourth and sixth order terms are obtained with chiral invariant couplings of the mesons rho and omega fields to the non linear sigma model making the limit of their masses going to infinity. This is possible with a constant fixed pion decay constant, $f_{\pi}=\eta$, which, in the vacuum, is equal to the classical scalar field of the Linear sigma model (and to the chiral radius) but may not be inside the hadrons [4]. The winding number of the topological soliton is identified to the baryonic number (topologically conserved) of the resulting soliton, which is thus interpreted as a baryon. The solitonic character is responsible for the stability of such extended configuration.

The simplest usual Skyrmion Lagrangian density will be considered firstly as proposed by Skyrme [13] with the Hedgehog ansatz (given by $\boldsymbol{\pi}=\boldsymbol{r}$ ) for the invariant function: $U(\boldsymbol{r})=\cos (F(\boldsymbol{r}))+i \boldsymbol{\tau} \cdot \hat{\boldsymbol{\pi}} \sin (F(\boldsymbol{r}))$. The coupling constant $e$ may be related to the pion-pion scattering coupling [15]. The values usually adopted for this coupling ( $e \simeq 4-6$ ) are also found in large $N_{c}$ analysis with extrapolation to $N_{c}=3$ using sum rules of [16] $\left(e \simeq(7.6-12) / \sqrt{N_{c}}\right)$. Its value is usually considered to be independent of $f_{\pi}$.

\subsection{Coupling a scalar sigma field: the modi- fied Skyrmion}

The "natural" way of imposing a coupling of a scalar meson field (such as the chiral partner of the pion) to the Skyrmion model is to consider the linear realization of the sigma model [17]. The scalar degree of freedom may be represented by a dynamical/variable scalar field, $\eta(r)$, which becomes the (new) scalar dynamical degree of freedom [3, 4]. This is equivalent to make the chiral radius dependent on the spatial coordinate due to the chiral symmetry. In this case one is led to the following replacement for the quadratic Skyrmion Lagrangian term:

$$
U(\boldsymbol{r}) \rightarrow \frac{\eta(\boldsymbol{r})}{f_{\pi}} U(\boldsymbol{r})
$$

A similar model had been considered previously [8] with basically the same numerical results. This replacement leads to the linear sigma model in the quadratic kinetic term. The potential term is introduced to complete the linear sigma model Lagrangian in the limit in which it becomes the $\lambda \sigma^{4}$ model which presents a potential with the spontaneous breakdown of symmetry. In the fourth order Skyrme term yields ambiguous results which are scale non-invariant Lagrangian terms and unstable solutions in the usually considered physical region for the parameters of the model $[3,4]$. Therefore it was not considered. The value of the condensate in the vacuum is found by the minimization of its potential. In the vacuum (as well as the presence of hadron(s)) the chiral radius as a dynamical variable has already been considered in $[4,11,18,7]$. In the spatially homogeneous case of $\eta(r)=f_{\pi}$ (constant), the usual Skyrmion equation and solutions are obtained in the infinite sigma mass limit. A complete account of solutions for these equations will be shown elsewhere. The boundary conditions for the respective Euler Lagrange equations are discussed in [4]. The "quanta" of $\eta$ field may be expected to correspond to the lightest scalarisoscalar meson, the sigma. It is expected to be a (very) broad isoscalar resonance in the s-wave of low energy $\pi-\pi$ scattering and other processes, with a mass in the range 500$800 \mathrm{MeV}[5,6,3,4]$.

\subsection{Magnetic Moments}

The magnetic moments $\left(\mu_{i}\right)$ of the proton and neutron are calculated as the averaged value of the operator:

$$
\mu_{i}=\epsilon_{i j k} r^{j}\left(\frac{1}{2} B^{k}+J_{V^{(3)}}^{k}\right),
$$

where $B^{k}$ and $J_{V(3)}^{k}$ are the isoscalar baryonic current and the third component of the isovector current respectively. In the usual Skyrmion model [19] the proton and neutron $\mu$ yields respectively:

$$
\begin{aligned}
& \mu_{p}=2 M_{N}\left\{\frac{1}{12 \mathcal{I}}<r^{2}>_{E, 0}+\frac{\mathcal{I}}{6}\right\}, \\
& \mu_{n}=2 M_{N}\left\{\frac{1}{12 \mathcal{I}}<r^{2}>_{E, 0}-\frac{\mathcal{I}}{6}\right\} \text {, }
\end{aligned}
$$

where: $M_{N}$ is the nucleon mass

$$
\begin{aligned}
& \mathcal{I}=4 \pi \int d r r^{2} \frac{s^{2}}{6}\left\{4 \eta(r)^{2}+\frac{4}{e^{2}}\left(F^{\prime 2}+\frac{s^{2}}{r^{2}}\right)\right\} \\
& <r^{2}>_{E, 0}=-\frac{2}{\pi} \int d r r^{2} s F^{\prime},
\end{aligned}
$$

which are the moment of inertia of the (quantized) rotating soliton and the averaged squared isoscalar electric radius respectively [19].

\subsection{Numerical results and discussion}

For several values of the pion mass $\left(m_{\pi}=\right.$ $0,139,200,400,600,700,800 \mathrm{MeV})$, the magnetic moments of the neutron and of the proton were calculated for both Skyrmion models discussed above. For the extended Skyrmion model (Skyrmignon, with the scalar field) two calculations were done with expressions (3): 1) with the observed nucleon mass (nearly $M_{N} \simeq 940 \mathrm{MeV}$ ) and 2) 


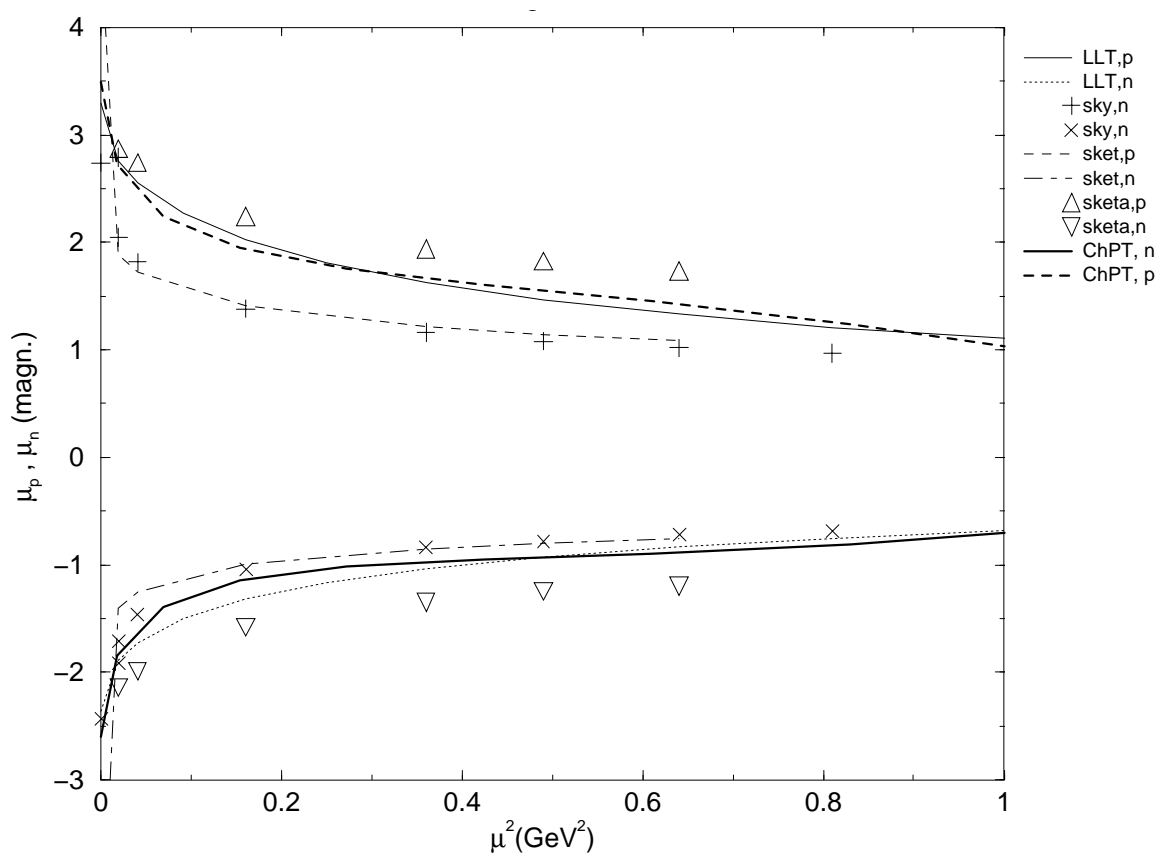

Figure 1. Magnetic moments of the neutron and of the proton as functions of the squared pion mass $\left(\mathrm{GeV}^{2}\right)$. Full (dotted) line for the proton (neutron) $\mu$ proposed by L.L.T. [1]; plus + (times $\times$ ) obtained from the usual Skyrmion model for $\mu_{n}\left(\mu_{p}\right)$; dashed (short-long dashed) lines obtained from the extended Skyrmion model [4] using $M_{N}=940 \mathrm{MeV}$; up triangles $\Delta$ (down triangles $\nabla$ ) obtained from the extended Skyrmignon model [4] using the nucleon mass from the model itself, $\left(M_{N} \simeq 1250 \mathrm{MeV}\right)$. Thick dashed and solid lines are the fit found with chiral perturbation theory at one loop [2].

with the mass resulting from the topological soliton numerical solution $[19,4]$.

We compare the results for the magnetic moments with different pion masses obtained from the calculations with the Skyrmion and extended Skyrmion models with those obtained from 1)the cloudy bag model [1] and 2) chiral perturbation theory (CPT) [2] which are used for extrapolations in Lattice calculations [1]. Several resulting points and curves are shown and compared in Figure 1.

The parameterization found out and proposed in [1] for the dependence of the magnetic moment on the pion mass using the Cloudy Bag Model is given by:

$$
\mu_{i}=\frac{A_{i}}{1+B_{i} m_{\pi}+C_{i} m_{\pi}^{2}},
$$

where $i=n, p$ distinguishes the neutron from the proton, $A, B, C$ are coefficients found by fitting the points from calculations shown in Fig. 1.

The results obtained with the Skyrmion and the extended Skyrmion ( coupled to the sigma) models are similar to those of the CBM and CPT. This may bring a certain credibility to both results but there is no an unique desirable possible extrapolation which therefore depends on the physical input of the effective model. In particular we notice that although the behavior (slopes) of all the curves are similar, for realistic pion mass the resulting values for the magnetic moment may be quite different, mainly of the proton. All the calculations done, shown in the Fig. 1, yield points which can be nearly fitted with expression (6). However the coefficients are somewhat different for each calculation and there seems to exist other extrapolations which may fit the points.

\section{Acknowledgements}

F.L.B. was supported by FAPESP, Brazil. The author also thanks M.R. Robilotta for several discussions about the Skyrmion model and M. Nielsen for suggesting the calculation of large pion/quark masses.

\section{References}

[1] D.B. Leinweber, D.H. Lu, and A.W. Thomas, Phys. Rev. D60, 034014 (1999).

[2] M. Göckeler et al., hep-lat/0303019; T.R. Hemmert and W. Weise, Eur. Phys. Journ. A15, 487 (2002); T.R. Hemmert and W. Weise, hep-lat/0303019.

[3] F. L. Braghin, M.Sc. thesis, unpublished, Brazil, 1992.

[4] F. L. Braghin and I.P. Cavalcante, Phys. Rev. C65 065207 (2003); F.L. Braghin, work presented in Reunião de Trabalho de Física Nuclear no Brasil, Itatiaia, RJ, Brasil (1998), without proceedings.

[5] N.A. Tornqvist and M. Roos, Phys. Rev. Lett. 76, 1575 (1996).

[6] E.M. Aitala et al, Phys. Rev. Lett. 86, 770 (2001).

[7] B.R. Martin, D. Morgan, and G. Shaw, Pion-Pion Interaction in Particle Physics, Acad. Press, (1976); K. Hagiwara et al Particle Data Group Collaboration, Phys. Rev. D 66, 010001 (2002).

[8] T. Kunihiro et al, hep-lat/0210012; U.G.-Meissner, R. Johnson, N.W. Park, and J. Schechter, Phys. Rev. D37, 1285 (1988). 
[9] F.L. Braghin, under preparation; pre-print IF-USP, (2001/2003); hep-ph/0206107; submitted to publication; seminar in Journey on Bosons, Gauge Symmetries and related subjects, Instituto de Física, USP, São Paulo, Brazil, 2002 ,

http://www.axpfepl.if.usp.br/ finpe/workshop/.

[10] Chiral radius discussions: F.L. Braghin and M.R. Robilotta, M.Sc. thesis, Brazil, unpublished, (1992).

[11] I. P. Cavalcante, M. R. Robilotta, Phys. Rev. C63 044008 (2001).

[12] V. Timoteo and C.L. Lima, Phys. Lett. B448 (1) 448 (1999); Mod. Phys. Lett. A15, 219 (2000); F.L. Braghin, C.L. Lima, V. Timoteo, and M.R. Robilotta, under preparation.

[13] T.H.R. Skyrme, Proc. Roy. Soc. Ser. A260 127 (1961).
[14] O.L. Battistel, Doctoral thesis, USP, São Paulo, Brazil, 1994.

[15] T.N. Pham, T.N. Truong, Phys. Rev. D31, 3027 (1985); M. Mashaal, T.N. Pham, and T.N. Truong, Phys. Rev. D34, 3484 (1986). J.F. Donoghue, E. Golowich, and B.R. Holstein, Phys. Rev. Lett. 53, 747 (1984).

[16] H.G. Dosch, S. Narison, Phys. Lett. B184, 78 (1987).

[17] M. Gell-Mann and M. Lévy, Nuovo Cimento 16, 705 (1960).

[18] F.L. Braghin, pre-print, IF-USP, São Paulo, SP, submitted to publication, 2001.

[19] G.S. Adkins, in Chiral Solitons, ed. by G.S. Adkins, Add.Wesley, 1988; O.L. Battistel, Doctoral thesis, Instituto de Física da Universidade de São Paulo, Brazil, 1994. 